\title{
High incidence of major bleeding with off-label use of edoxaban
}

\author{
Rika Fukui,, Daiki Hira, ${ }^{1,2}$ Yuki Kunitsu, ${ }^{1}$ Tetsuichiro Isono, ${ }^{1}$ Yohei Tabuchi, ${ }^{1}$ Yoshihiro Ikuno, ${ }^{1,3}$ Satoshi Ueshima, ${ }^{2}$ \\ Hideki Itoh, ${ }^{4,5}$ Toshihiro Tanaka, ${ }^{3,6}$ and Tomohiro Terada ${ }^{1, *}$ \\ ${ }^{1}$ Department of Pharmacy, ${ }^{3}$ Medical Safety Section, ${ }^{5}$ Department of Cardiovascular Medicine, and ${ }^{6}$ Department of Dermatology, Shiga University of Medical \\ Science Hospital, Seta Tsukinowa-cho, Otsu, Shiga 520-2192, Japan \\ ${ }^{2}$ College of Pharmaceutical Sciences, Ritsumeikan University, 1-1-1 Noji-higashi, Kusatsu, Shiga 525-8577, Japan \\ ${ }^{4}$ Division of Patient Safety, Hiroshima University Hospital, Kasumi Minami-ku, Hiroshima 734-8551, Japan
}

(Received 18 January, 2021; Accepted 15 March, 2021)

In clinical practice, edoxaban is sometimes prescribed for off-label use based on the hypothesis that it is as safe and effective as warfarin. However, there is limited safety information on off-label use due to lack of clinical trial. We aimed to analyze the tolerability of off-label use of edoxaban and to identify patient characteristics associated with major bleeding as adverse effects. Patients under edoxaban treatment between January 2017 and December 2017 were enrolled in this retrospective cohort study. The incidence of major bleeding with off-label use compared with on-label use was analyzed using by log-rank test. Univariate and multivariate regression analysis were undertaken to detect independent variables with significant odds ratio that associated with major bleeding. After the exclusion criteria were applied, the patients were divided into two groups: off-label group $(n=30)$ and on-label group $(n=161)$. Incidence of major bleeding was found to be higher in the off-label group (13.3\%) than in the on-label group $(3.7 \%)(p<0.05)$. Multivariate adjustment showed that the off-label use or portal vein thrombosis and patients with history of major bleeding has significantly higher incidence of major bleeding. We demonstrated that off-label use of edoxaban may be a significant risk factor for major bleeding.

Key Words: direct oral anticoagulants, edoxaban, anticoagulants, off-label, bleeding

I $\mathrm{n}$ Japan, the first direct oral anticoagulant (DOAC), dabigatran etexilate, was approved for ischemic stroke and systemic embolism prevention in patients with nonvalvular atrial fibrillation (NVAF) in 2011. Until the advent of DOACs, warfarin, a vitamin $\mathrm{K}$ antagonist, was the only available oral anticoagulant. Warfarin and other vitamin $\mathrm{K}$ antagonists are highly effective treatments that reduce the risk of stroke by approximately two thirds. ${ }^{(1)}$ However, warfarin has some limitations: a narrow therapeutic range, drug and food interactions, variable/ unpredictable pharmacokinetic/pharmacodynamic properties, and the need for regular coagulation monitoring.(2) DOACs were intracranial and fatal bleeding occurred lesser frequently than warfarin in phase III trials, ${ }^{(3-6)}$ but the number of on-label indication is very limited. Warfarin is approved for various types of thromboembolism, but DOACs are only approved for indications such as the prevention of ischemic stroke and systemic embolism in patients with NVAF, treatment and prevention of recurrence of venous thromboembolism (VTE) and pulmonary embolism (PE), and prevention of VTE in people undergoing elective hip or knee replacement surgery or hip fracture repair in Japan. Thus, warfarin may be only available oral option for some anticoagulant therapy. Several reports have demonstrated the effectiveness of off-label use of DOACs for left ventricular thrombosis, Trousseau syndrome, and portal vein thrombosis (PVT). ${ }^{(7-11)}$ However, most of these reports were a small number of patients, the safety of DOACs in off-label use remains uncertain. In on-label use, the FUSHIMI AF registry showed that the incidence rate of major bleeding in patients with NVAF was $1.8 \%$ per year. ${ }^{(12)}$ Only a few clinical data are available concerning safe off-label use of DOACs; hence incidence of major bleeding for off-label use has been unknown. Moreover, thrombosis is the most frequent complication and the second leading cause of death in cancer patients. ${ }^{(13,14)}$ Cancer patients often develop PVT and Trousseau syndrome as complications; however, DOACs have not been recommended or only part of DOACs were positioned as optional per guidelines. ${ }^{(15-17)}$ In the Hokusai VTE Cancer study, edoxaban was shown noninferior to dalteparin with respect to the composite outcome of recurrent VTE or major bleeding. ${ }^{(18)}$ Edoxaban is easier to control than warfarin in that it is not affected by drug and food and has immediate expression of the anticoagulant effect after administration because edoxaban selectively inhibits factor Xa. Therefore, edoxaban may be used in cancer patients and it is highly advisable to assess the tolerability of edoxaban for off-label use in clinical practice.

In this study, we aimed to analyze the safety and tolerability of off-label edoxaban use and to identify factors associated with major bleeding.

\section{Materials and Methods}

Patient selection criteria. A retrospective cohort study was conducted using data from a single hospital in Japan. Medical records of patients who were prescribed with edoxaban for the year 2017 in Shiga Universal of Medical Science Hospital were reviewed. The patients prescribed edoxaban for prevention of ischemic stroke and systemic embolism in patients with NVAF, treatment and prevention of recurrence of VTE and PE were defined as on-label group. Off-label use was defined by the absence of an approved indication in the package insert. Exclusion criteria were as follows: (1) patients with unknown indication, (2) patients who underwent orthopedic surgery because of short-term medication, (3) patients with inadequate background information, (4) patients who did not visit our hospital after prescription, (5) patients who did not take edoxaban due to a switch to other anticoagulant after prescription, and (6) patients who were taking edoxaban before admission.

*To whom correspondence should be addressed. E-mail: teradat@belle.shiga-med.ac.jp 
Ethics approval statement. This study was conducted in accordance with the "Declaration of Helsinki", and approved by the Ethics Board of Shiga University of Medical Science (Approval Number: 30-081). Off-label use in this study was approved by the Intractable Diseases Treatment Technology and Unapproved Medication Assessment Management Office of Shiga University of Medical Science Hospital. Written informed consent was waived because of the anonymous nature of the data. As an ethical consideration, participants had been provided the opportunity to opt out of this research based on the written information about using electronic records for this research which was posted in the homepage of the Shiga University of Medical Science Hospital.

Primary outcome. The primary outcome was the incidence of major bleeding in a year after edoxaban prescription. All patients were followed at least a year after taking edoxaban at a first time. The incidence rates of major bleeding between the off-label group and on-label group were compared. The factors related to major bleeding were analyzed using multivariate analysis. Major bleeding in multivariate analysis included all observation periods. Major bleeding was defined according to the International Society on Thrombosis and Hemostasis (ISTH) bleeding criteria. ${ }^{(19)}$ ISTH major bleeding was defined as follows: (i) fatal bleeding, and/or (ii) symptomatic bleeding in a critical area or organ, such as intracranial, intraspinal, intraocular, retroperitoneal, intra-articular or pericardial, or intramuscular with compartment syndrome, and/or (iii) bleeding causing a fall in hemoglobin levels of $20 \mathrm{~g} / \mathrm{L}(1.24 \mathrm{mmol} / \mathrm{L})$ or more or leading to a transfusion of $2 \mathrm{U}$ or more of whole blood or red blood cells.

The HAS-BLED score for major bleeding risk $^{(20,21)}$ was used as explanatory variables for multivariate analysis, which includes renal dysfunction, liver dysfunction, stroke history, use of antiplatelet drugs, use of nonsteroidal anti-inflammatory drugs (NSAIDs), heart failure, hypertension, diabetes, major bleeding history or predisposition, and vascular disease. The factors for labile international normalized ratio (INR) and alcohol concomitantly were not included as explanatory variables because there was no participant who applicable for these factors. In addition, gastric secretion inhibitors (proton pump inhibitors or histamine-2 receptor antagonists) presumed to control gastric bleeding and malignant tumors presumed as a risk factor for bleeding were also added as explanatory variables. Renal dysfunction was defined as maintenance dialysis and/or kidney transplant and/or creatinine level $(\mathrm{Cr})>2.26 \mathrm{mg} / \mathrm{dl}$. Liver dysfunction was defined as chronic liver disease, total bilirubin level (T-Bil) $\geq 2 \mathrm{x}$ upper limit of normal, or aspartate aminotransferase (AST)/alanine aminotransferase (ALT)/alkaline phosphatase (ALP) $\geq 3 \mathrm{x}$ upper limit of normal. Major bleeding history or predisposition was defined as major bleeding based on ISTH bleeding criteria. Heart failure was defined as a diagnosis of congestive heart failure by the attending physician or an ejection fraction $(\mathrm{EF}) \leq 50 \%$. Hypertension was defined as high blood pressure with systolic blood pressure $\geq 160 \mathrm{mmHg}$ or history of or ongoing oral antihypertensive medication use. Diabetes was defined as hemoglobin A1c (HbAlc) $\geq 7.0 \%$ or history of or ongoing hypoglycemic medication use. Vascular disease was defined as a history of myocardial infarction, peripheral arterial disease, and aortic plaque.

Statistical analysis. To analyze the averages of continuous variables of patient characteristics and the proportions of categorical variables of patient characteristics between the groups, unpaired $t$ test and Fisher's exact test were used, respectively. The difference in edoxaban daily doses was compared between the two groups using Mann-Whitney $U$ test. The probability of event-free survival was assessed by Kaplan-Meier method and log-rank test. Univariate and multivariate logistic regression analyses were performed to identify independent predictors of the endpoint. The results of logistic regression analyses were expressed as odds ratio and 95\% confidence interval $(95 \% \mathrm{CI})$. In all analyses, $p$ values were two-tailed and $p<0.05$ was considered as statistically significant. Statistical analyses were performed using SPSS ver. 22 (SPSS Inc., Chicago, IL) and R ver. 3.0.2 (The R Foundation for Statistical Computing, Vienna, Austria).

\section{Results}

Background. A total 328 patients were enrolled in this study. After the exclusion criteria were applied, 191 patients were selected (Fig. 1). A total of 30 patients were included in the off-label group and 161 patients in the on-label group. The demographics and baseline clinical characteristics of the 191 patients were presented in Table 1. Significantly lower age, lower stroke risk score $\left(\mathrm{CHADS}_{2}\right.$ and $\mathrm{CHA}_{2} \mathrm{DS}_{2}$-VASc), and higher ratio of malignant tumor were compared between the off-label group and on-label group. Sixty percent (18 of 30) of patients in the off-label group were diagnosed with PVT. In both on-label and off-label groups, edoxaban doses were appropriately reduced based on renal function, body weight, and concomitant drugs as described in the package insert. Only one case in the on-label group showed an overdose $(60 \mathrm{mg})$, albeit the recommended dose of $30 \mathrm{mg}$ for low body weight $(<60 \mathrm{~kg})$.

Comparison of the bleeding risk. A follow-up period of 12 months showed a significantly higher incidence of major bleeding in the off-label group (4 out of 30,13.3\%) than in the on-label group ( 6 out of $161,3.7 \%)(\mathrm{OR}=4.22,95 \%$ CI 1.38 $12.93, p<0.05$ by univariate logistic regression analysis). Gastrointestinal bleeding was observed in 3 patients and hematuria in 1 patient in the off-label group. And gastrointestinal bleeding was observed in 2 patients, genital bleeding in 1 patient and 3 patients were transfused with no apparent bleeding source in the on-label group. Kaplan-Meier curves for the time to major bleeding event indicated significantly higher incidence rates in the off-label group than in the on-label group $(p<0.05$ by log-rank test), as shown in Fig. 2.

Multivariate analysis of the risk factors for major bleeding. Table 2 presents univariate and multivariate logistic regression analyses associated with major bleeding. Univariate analysis showed that the clinical risk factors for major bleeding were off-label use $(\mathrm{OR}=4.22,95 \%$ CI 1.38-12.93, $p<0.05)$, PVT $(\mathrm{OR}=9.11,95 \%$ CI 2.78-29.88, $p<0.01)$, liver dysfunction $(\mathrm{OR}=3.50,95 \%$ CI $1.09-11.20, p<0.05)$, and major bleeding history $(\mathrm{OR}=10.75,95 \%$ CI $2.16-53.63, p<0.01)$. Because off-label use was significantly correlated with PVT, multicollinearity should be considered in the multivariate analysis. Therefore, two independent multivariate logistic regression analyses with off-label use or PVT were performed. Multivariate logistic regression analysis with off-label use showed that off-label use $(\mathrm{OR}=4.37,95 \%$ CI $1.09-17.46, p<0.05)$ and major bleeding history $(\mathrm{OR}=12.38,95 \%$ CI $2.14-71.50, p<0.01)$ were detected as the independent risk factors for major bleeding. Furthermore, multivariate logistic regression analysis with PVT showed that the independent risk factors for major bleeding were PVT $(\mathrm{OR}=23.77,95 \%$ CI 3.28-172.15, $p<0.01)$ and major bleeding history $(\mathrm{OR}=17.27,95 \% \mathrm{CI} 2.74-108.64, p<0.01)$. The OR values for renal dysfunction, antiplatelet drugs, NSAIDs, heart failure, and vascular disease were not able to be calculated because of their small population and low incidence of events.

\section{Discussion}

After multivariate adjustment, off-label use or diagnosis of PVT and major bleeding history were detected as the significant risk factors of major bleeding caused by edoxaban treatment, in which major bleeding history was a well-known risk factor. ${ }^{(20-22)}$ It is the first report that showed off-label use of edoxaban as a 


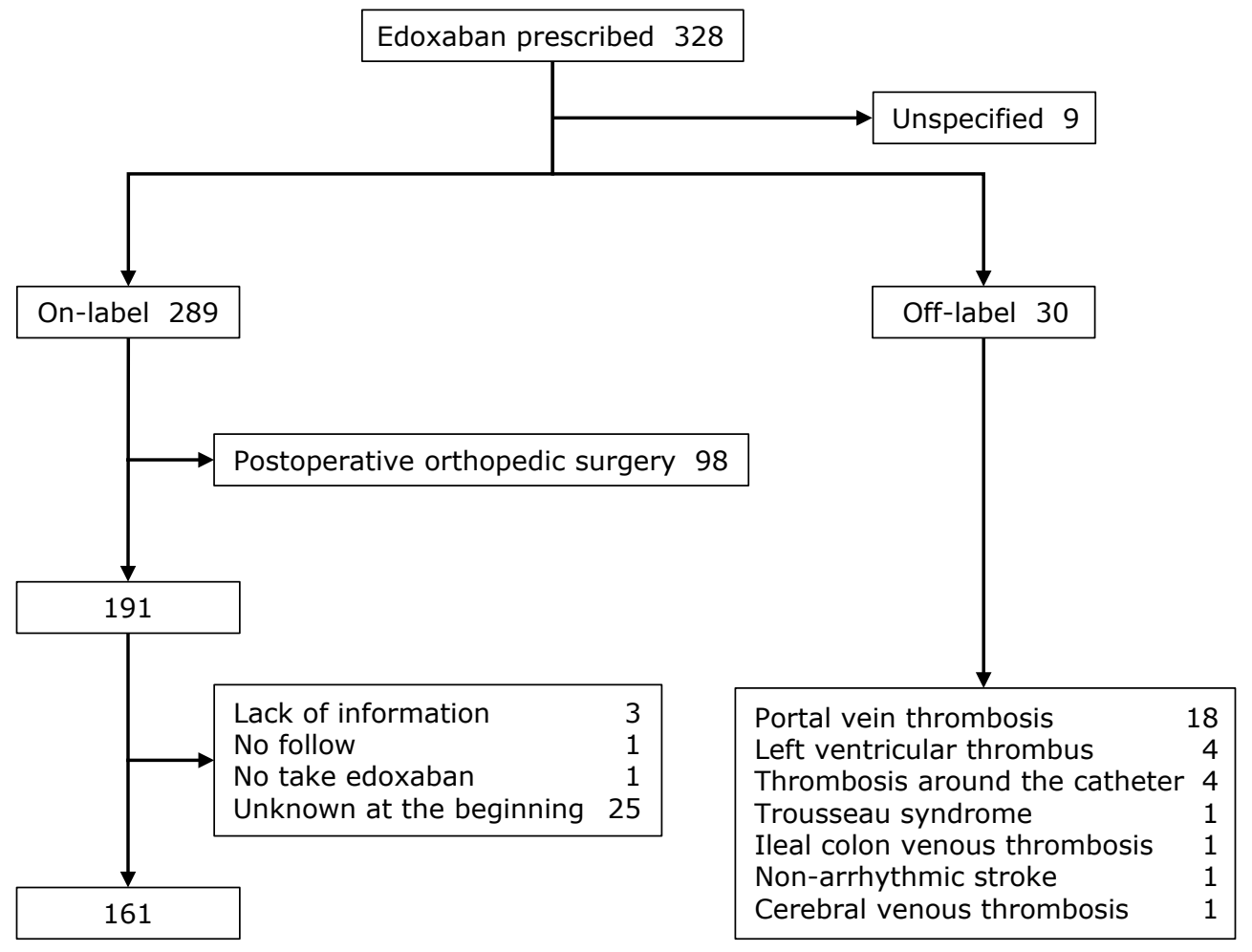

Fig. 1. Patient selection criteria.

Table 1. Patient background

\begin{tabular}{|c|c|c|c|}
\hline & Off-label $(n=30)$ & On-label $(n=161)$ & $p$ value \\
\hline Sex (male/female) & $18 / 12$ & $89 / 72$ & 0.692 \\
\hline Age (years) & $63.6 \pm 9.7$ & $70.4 \pm 10.3$ & $<0.01 * *$ \\
\hline Body weight $(\mathrm{kg})$ & $55.3 \pm 12.9$ & $58.6 \pm 12.1$ & 0.169 \\
\hline Body surface area $\left(\mathrm{m}^{2}\right)$ & $1.6 \pm 0.2$ & $1.6 \pm 0.2$ & 0.308 \\
\hline Uncorrected eGFR (ml/min) & $73.6 \pm 18.9$ & $67.4 \pm 22.6$ & 0.164 \\
\hline $\mathrm{CHADS}_{2}$ score & $1.2 \pm 1.0$ & $2.0 \pm 1.7$ & $<0.01 * *$ \\
\hline $\mathrm{CHA}_{2} \mathrm{DS}_{2}$-VASc score & $2.0 \pm 1.1$ & $2.8 \pm 1.7$ & $<0.01 * *$ \\
\hline HAS-BLED score & $1.6 \pm 1.1$ & $1.7 \pm 1.0$ & 0.644 \\
\hline Malignant tumor (yes/no) & $19 / 11$ & $68 / 93$ & $0.045^{*}$ \\
\hline Administration period (days) & $323.8 \pm 427.9$ & $395.8 \pm 384.5$ & 0.356 \\
\hline Gastric secretion inhibitors (PPI/H2RA/no) & $21 / 1 / 8$ & $91 / 8 / 62$ & 0.388 \\
\hline Daily dose & & & 0.361 \\
\hline $15 \mathrm{mg}$ & 1 & 5 & \\
\hline $30 \mathrm{mg}$ & 24 & 116 & \\
\hline $60 \mathrm{mg}$ & 5 & 40 & \\
\hline Indication & & & - \\
\hline $\begin{array}{l}\text { Prevention of ischemic stroke and systemic } \\
\text { embolism in patients with NVAF }\end{array}$ & 0 & 92 & \\
\hline $\begin{array}{l}\text { Treatment and prevention of recurrence of } \\
\text { VTE and PE }\end{array}$ & 0 & 69 & \\
\hline PVT & 18 & 0 & \\
\hline Left ventricular thrombus & 4 & 0 & \\
\hline Thrombosis around the catheter & 4 & 0 & \\
\hline Trousseau syndrome & 1 & 0 & \\
\hline Ileocolic venous thrombosis & 1 & 0 & \\
\hline Non-arrhythmic stroke & 1 & 0 & \\
\hline Cerebral venous thrombosis & 1 & 0 & \\
\hline
\end{tabular}




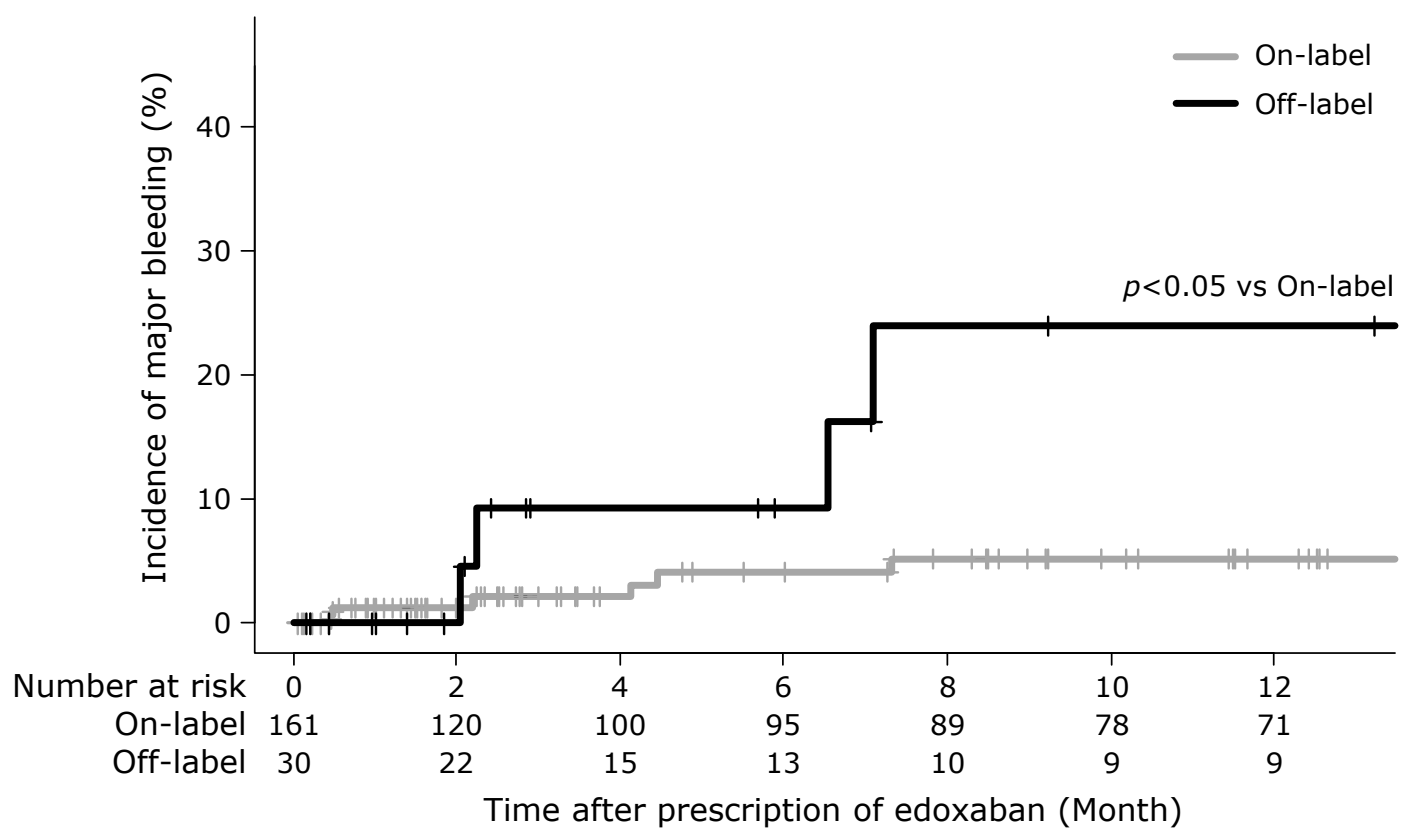

Fig. 2. Kaplan-Meier curve for the time to major bleeding event. Off-label group (black line) vs on-label group (gray line). Tick marks mean censored cases.

Table 2. Univariate and multivariate analyses of the risk factors for major bleeding

\begin{tabular}{lcc}
\hline \multicolumn{1}{c}{ Covariate } & $\begin{array}{c}\text { Univariate analysis } \\
\text { OR }(95 \% \mathrm{Cl})\end{array}$ & $\begin{array}{c}\text { Multivariate analysis } \\
\text { OR }(95 \% \mathrm{Cl})\end{array}$ \\
\hline Off-label use & $4.22(1.38-12.93)$ & $4.37(1.09-17.46)$ \\
Age per 1 year increase (year) & $0.98(0.93-1.03)$ & - \\
Male Sex & $1.63(0.54-4.96)$ & - \\
Body weight per $1 \mathrm{~kg}$ increase $(\mathrm{kg})$ & $0.99(0.94-1.03)$ & - \\
Uncorrected eGFR per 1 $\mathrm{ml} / \mathrm{min}$ increase $(\mathrm{ml} / \mathrm{min})$ & $1.00(0.97-1.02)$ & $1.25(0.29-5.37)$ \\
Liver dysfunction & $3.50(1.09-11.20)$ & - \\
Hypertension & $0.70(0.24-2.00)$ & - \\
Diabetes & $0.73(0.20-2.70)$ & - \\
Stroke & $1.08(0.29-4.06)$ & $12.38(2.14-71.50)$ \\
Major bleeding history & $10.75(2.16-53.63)$ & - \\
HAS-BLED score per 1 point increase & $1.19(0.70-2.02)$ & - \\
Malignant tumor & $1.40(0.49-4.04)$ & - \\
Gastric secretion inhibitors & $1.52(0.59-3.91)$ & - \\
\hline
\end{tabular}

$\mathrm{Cl}$, confidence interval; eGFR, estimated glomerular filtration rate; $\mathrm{OR}$, odds ratio.

risk factor for the major bleeding.

Not limit to edoxaban, off-label prescribing is common in clinical practice, in cancer patients ranged from $18 \%$ to $41 \%{ }^{(23)}$ Off-label prescribing remains an issue of controversy, due to uncertainty around the clinical benefits and potential toxicities, limited evidence to support clinical decision-making, increased out-of-pocket costs for patients and ethical concerns around the lack of informed consent. Most clinicians perceive off-label prescribing as appropriate and believe that the benefits typically outweigh any risks. ${ }^{(24)}$ However, there was 1.44 times greater risk of occurrence of adverse events associated with the use of off-label drugs. ${ }^{(25)}$ The use of off-label with the insufficient information may lead to several problems including adverse effects and an increased risk/benefit balance. In the present study, off-label use of edoxaban was also associated with a higher risk of major bleeding events $(\mathrm{OR}=4.37,95 \%$ CI $1.09-17.46$, $p<0.05$ )

Despite these limited indications, DOACs are widely prescribed off-label for various other thrombosis.(26) Because warfarin is limited by the narrow therapeutic range, the need for monitoring, and food and drug interactions. ${ }^{(2)}$ However, DOACs have not yet found mainstream success as an alternative for thrombosis treatment of various areas. The American College of Chest Physicians (ACCP), American Society of Clinical Oncology (ASCO), and National Comprehensive Cancer Network (NCCN) clinical practice guideline in oncology suggest DOACs are generally not recommended or only part of DOACs were positioned as optional for patients with VTE due to a lack of large-scale studies and the absence of clinical experience with the use of DOACs. ${ }^{(15-17)}$ The limitation of a specific reversal 
agent of edoxaban has caused concern among clinicians because anticoagulant reversal may be required in specific clinical situations, such as in patients experiencing spontaneous or traumatic bleeds, or in anticoagulated patients requiring emergency surgery or other invasive procedures. ${ }^{(26)}$ Furthermore, the system called "the relief system for sufferers from adverse drug reactions" to provide various relief benefits and prompt relief to patients and their families, apart from civil liabilities, in relation to health damage caused by adverse reactions in spite of proper use of drugs in Japan cannot be applied to the side effect of off-label use. (27) Although several studies reported that DOACs are safe and efficacious alternatives to traditional anticoagulation for off-label, literature evaluating DOACs use in the treatment of off-label is sparse. ${ }^{(7-11)}$ Hence, the risks and benefits of off-label use have to be assessed, and patients have to be informed accordingly when licensed alternative treatments are lacking.

Several studies reported the safety of drug administration for PVT patients, ${ }^{(9-11)}$ but this study suggested that off-label use of edoxaban, especially for PVT patients, associated with high incidence of major bleeding. PVT is common in patients with advanced liver disease and malignancy, and complicated by bleeding from a gastroesophageal variceal and an imbalance of the whole coagulation system associated with liver dysfunction or malignancy. ${ }^{(13,14,28)}$ And patients with PVT may have higher exposure to a high concentration of edoxaban due to severe liver dysfunction because approximately $50 \%$ of systemically absorbed edoxaban is eliminated via hepatic metabolism and biliary secretion. ${ }^{(29)}$ An ENGAGE AF-TIMI 48 subanalysis concluded a history of liver disease did not alter the relative efficacy and safety of edoxaban compared with warfarin. ${ }^{(30)}$ But bleeding was increased in patients with liver disease, ${ }^{(30)}$ and it was known that increasing plasma trough edoxaban concentrations were associated with a higher risk of major gastrointestinal bleeding. ${ }^{(22)}$ As the risk of bleeding is uncertain, care should be taken during treatment with edoxaban to diagnose and treat any potential bleeding, especially gastrointestinal bleeding.

Major bleeding history is associated with an increased risk of hemorrhage. ${ }^{(20-22)}$ The recommended dose of edoxaban stated on its package insert is dependent on only body weight, renal function, and concomitant drugs that interact with edoxaban via P-gp. ${ }^{(31)}$ However, it was known that genetic polymorphisms in drug-metabolizing enzymes and drug transporters affect the pharmacokinetics of DOACs. ${ }^{(32,33)}$ A genome-wide association study of dabigatran identified that a polymorphism of CES1 was associated with low exposure to active metabolite of dabigatran. $^{(34)}$ Similar to other DOACs, pharmacokinetics of edoxaban is regulated by metabolic enzymes and transporters such as CES1, CYP3A4/5, and ABCB1. Patients with major bleeding history taking edoxaban on the package insert may have been higher exposure to a high concentration due to pharmacokinetic variability. ${ }^{(32)}$ Due to several factors underlying major bleeding history, pharmacogenetics (PGx) may provide useful information for individualized edoxaban pharmacotherapy to prevent the risk of adverse reactions.

Some limitations of the study should be acknowledged. First, there are differences in baseline patient characteristics, the indications for anticoagulation. This study was adjusted multiple variables between two group by multivariate analysis, because this study compared totally different two group. The second major limitation is the quality of the study. The retrospective collection of data does not allow exclusion of any potential confounding factor escaping our analysis. There was not controlled study as the anticoagulation prescribing (initiation, choice of the agents, discontinuation, and transitioning to and from traditional anticoagulants due to intolerance or adverse effect) was not under the investigators' control. Finally, we are inherently limited by the number of patients in our study cohort. Because of limited patient number of the present retrospective study, it is difficult to absolutely divide the impact of PVT and off label use. In addition, a number of major bleeding in off-label group happened after 6 months from the start of edoxaban. Although progression of the primary disease may be an internal factor affecting major bleeding, the limited patient number of the present study could not define a causal relationship between progression of the primary disease and incidence of major bleeding on this research. Therefore, further large-scale clinical trial should be conducted to describe these impacts of PVT and onset time of major bleeding. However, our present study indicated that off-label use, including PVT, may be a risk factor of major bleeding. The risk of bleeding should probably be alerted of off-label use if one considers the high incidence of bleeding in our study. The risk/benefit ratio must be assessed carefully in individual patients.

We demonstrated that the off-label use of edoxaban, especially in PVT patients, increased the incidence of major bleeding. Off-label use is not illegal, and may sometimes be clinically appropriate. On this research, PVT patients with history of major bleeding should have sought alternative anticoagulants except for edoxaban. Patient using off-label edoxaban should be carefully selected. In the case of off-label use, patients have to be carefully considered to minimize patient harm and informed prior to the start of medication, after a thorough risk-benefit analysis. Accordingly, confirmation of risk factor and PGx tests may reduce the risk of major bleeding. Extensive research is needed to evaluate the relationship between PGx and bleeding risk for patients with off-label use of DOACs.

\section{Author Contributions}

All authors had access to the data and a role in writing this manuscript. RF, DH, YK, TI, YT, YI, SU, HI, TTa, and TTe contributed to the study conception and design. RF, DH, and SU were involved in the collection and analysis of data. RF drafted the manuscript, and DH, YK, TI, YT, YI, SU, HI, TTa, and TTe critically revised the manuscript. All authors read and approved the final manuscript.

\section{Acknowledgments}

This work was supported by JSPS KAKENHI Grant Number JP18K06782.

\section{Conflict of Interest}

No potential conflicts of interest were disclosed.

\section{References}

1 Hart RG, Benavente O, McBride R, Pearce LA. Antithrombotic therapy to prevent stroke in patients with atrial fibrillation: a meta-analysis. Ann Intern Med 1999; 131: 492-501.

2 Ansell J, Hirsh J, Hylek E, Jacobson A, Crowther M, Palareti G. Pharmacology and management of the vitamin K antagonists: American College of Chest Physicians Evidence-Based Clinical Practice Guidelines (8th edition).
Chest 2008; 133 (6 Suppl): 160S-198S.

3 Patel MR, Mahaffey KW, Garg J, et al. Rivaroxaban versus warfarin in nonvalvular atrial fibrillation. N Engl J Med 2011; 365: 883-891.

4 Connolly SJ, Ezekowitz MD, Yusuf S, et al. Dabigatran versus warfarin in patients with atrial fibrillation. N Engl J Med 2009; 361: 1139-1151.

5 Granger CB, Alexander JH, McMurray JJV, et al. Apixaban versus warfarin 
in patients with atrial fibrillation. $N$ Engl J Med 2011; 365: 981-992.

6 Giugliano RP, Ruff CT, Braunwald E, et al. Edoxaban versus warfarin in patients with atrial fibrillation. $N$ Engl J Med 2013; 369: 2093-2104.

7 Fleddermann AM, Hayes CH, Magalski A, Main ML. Efficacy of direct acting oral anticoagulants in treatment of left ventricular thrombus. $\mathrm{Am} \mathrm{J}$ Cardiol 2019; 124: 367-372.

8 Suzuki R, Nakamura Y, Miyazaki K, Ishii M, Shimokawa T, Okamoto H. A case report of a multi-cerebral infarction associated with trousseau syndrome before the diagnosis of ALK-positive lung adenocarcinoma. Japanese J Lung Cancer 2019; 59: 384-389.

9 Nagaoki Y, Aikata H, Daijyo K, et al. Efficacy and safety of edoxaban for treatment of portal vein thrombosis following danaparoid sodium in patients with liver cirrhosis. Hepatol Res 2018; 48: 51-58.

10 Hanafy AS, Abd-Elsalam S, Dawoud MM. Randomized controlled trial of rivaroxaban versus warfarin in the management of acute non-neoplastic portal vein thrombosis. Vascul Pharmacol 2019; 113: 86-91.

11 Intagliata NM, Henry ZH, Maitland $\mathrm{H}$, et al. Direct oral anticoagulants in cirrhosis patients pose similar risks of bleeding when compared to traditional anticoagulation. Dig Dis Sci 2016; 61: 1721-1727.

12 Yamashita Y, Uozumi R, Hamatani Y, et al. Current status and outcomes of direct oral anticoagulant use in real-world atrial fibrillation patients-Fushimi AF Registry-. Circ J 2017; 81: 1278-1285.

13 Timp JF, Braekkan SK, Versteeg HH, Cannegieter SC. Epidemiology of cancer-associated venous thrombosis. Blood 2013; 122: 1712-1723.

14 Khorana AA. Venous thromboembolism and prognosis in cancer. Thromb Res 2010; 125: 490-493.

15 Kearon C, Akl EA, Ornelas J, et al. Antithrombotic therapy for VTE disease: CHEST guideline and expert panel report. Chest 2016; 149: 315-352.

16 Key NS, Khorana AA, Kuderer NM, et al. Venous thromboembolism prophylaxis and treatment in patients with cancer: ASCO clinical practice guideline update. J Clin Oncol 2020; 38: 496-520.

17 Streiff MB, Holmstrom B, Bockenstedt PL, et al. Cancer-associated venous thromboembolic disease: NCCN clinical practice guidelines in oncology (NCCN guidelines) version 1.2020. The National Comprehensive Cancer Network. https://www.nccn.org/professionals/physician_gls/pdf/vte.pdf. Accessed 9 Jan 2021.

18 Raskob GE, van Es N, Verhamme P, et al. Edoxaban for the treatment of cancer-associated venous thromboembolism. N Engl J Med 2018; 378: 615624.

19 Schulman S, Kearon C; Subcommittee on Control of Anticoagulation of the Scientific and Standardization Committee of the International Society on Thrombosis and Haemostasis. Definition of major bleeding in clinical investigations of antihemostatic medicinal products in non-surgical patients. $J$ Thromb Haemost 2005; 3: 692-694.

20 Pisters R, Lane DA, Nieuwlaat R, de Vos CB, Crijns HJ, Lip GY. A novel user-friendly score (HAS-BLED) to assess 1-year risk of major bleeding in patients with atrial fibrillation: the Euro Heart Survey. Chest 2010; 138: 1093-1100.

21 Lip GY, Frison L, Halperin JL, Lane DA. Comparative validation of a novel risk score for predicting bleeding risk in anticoagulated patients with atrial fibrillation: the HAS-BLED (hypertension, abnormal renal/liver function, stroke, bleeding history or predisposition, labile INR, elderly, drug. J Am Coll Cardiol 2011; 57: 173-180.

22 Aisenberg J, Chatterjee-Murphy P, Friedman Flack K, et al. Gastrointestinal bleeding with edoxaban versus warfarin: results from the ENGAGE AF-TIMI 48 trial (effective anticoagulation with factor Xa next generation in atrial fibrillation-thrombolysis in myocardial infarction). Circ Cardiovasc Qual Outcomes 2018; 11: e003998.

23 Saiyed MM, Ong PS, Chew L. Off-label drug use in oncology: a systematic review of literature. J Clin Pharm Ther 2017; 42: 251-258.

24 Tan E, Stewart K, Pearce C, Chapman CB, Liaw ST. Understanding off-label and unlicensed prescribing: combining qualitative and quantitative methodologies. Clin Exp Pharmacol Physiol 2004; 31 (Suppl): A84.

25 Eguale T, Buckeridge DL, Verma A, et al. Association of off-label drug use and adverse drug events in an adult population. JAMA Intern Med 2016; 176: 55-63.

26 Priyanka P, Kupec JT, Krafft M, Shah NA, Reynolds GJ. Newer oral anticoagulants in the treatment of acute portal vein thrombosis in patients with and without cirrhosis. Int J Hepatol 2018; 2018: 8432781.

27 The relief system for sufferers from adverse drug reactions, etc. Ministry of Health Labour and Welfare. https://www.mhlw.go.jp/english/wp/wp-hw4/dl/ health_and_medical_services/P95.pdf. Accessed 2 Aug 2020.

28 Garcia-Tsao G, Bosch J. Management of varices and variceal hemorrhage in cirrhosis. N Engl J Med 2010; 362: 823-832.

29 Bathala MS, Masumoto H, Oguma T, He L, Lowrie C, Mendell J. Pharmacokinetics, biotransformation, and mass balance of edoxaban, a selective, direct factor xa inhibitor, in humans. Drug Metab Dispos 2012; 40: 22502255.

30 Qamar A, Antman EM, Ruff CT, et al. Edoxaban versus warfarin in patients with atrial fibrillation and history of liver disease. J Am Coll Cardiol 2019; 74: 179-189.

31 Krekels EH, Niebecker R, Karlsson MO, et al. Population pharmacokinetics of edoxaban in patients with non-valvular atrial fibrillation in the ENGAGE AF-TIMI 48 study, a phase III clinical trial. Clin Pharmacokinet 2016; 55 1079-1090.

32 Hira D, Terada T. BCRP/ABCG2 and high-alert medications: biochemical, pharmacokinetic, pharmacogenetic, and clinical implications. Biochem Pharmacol 2018; 147: 201-210.

33 Lentz SR. Genetic testing to guide warfarin dosing: impact of direct oral anticoagulants. Clin Pharmacol Ther 2016; 100: 128-130.

34 Paré G, Eriksson N, Lehr T, et al. Genetic determinants of dabigatran plasma levels and their relation to bleeding. Circulation 2013; 127: 1404-1412.

This is an open access article distributed under the terms of the Creative Commons Attribution-NonCommercial-NoDerivatives License (http://creativecommons.org/licenses/by-nc-nd/4.0/). 